\title{
PERSPECTIVES
}

OPINION

\section{Pathways to zoonotic spillover}

\section{Raina K. Plowright, Colin R. Parrish, Hamish McCallum, Peter J. Hudson, Albert I. Ko, Andrea L. Graham and James O. Lloyd-Smith}

Abstract | Zoonotic spillover, which is the transmission of a pathogen from a vertebrate animal to a human, presents a global public health burden but is a poorly understood phenomenon. Zoonotic spillover requires several factors to align, including the ecological, epidemiological and behavioural determinants of pathogen exposure, and the within-human factors that affect susceptibility to infection. In this Opinion article, we propose a synthetic framework for animal-to-human transmission that integrates the relevant mechanisms. This framework reveals that all zoonotic pathogens must overcome a hierarchical series of barriers to cause spillover infections in humans. Understanding how these barriers are functionally and quantitatively linked, and how they interact in space and time, will substantially improve our ability to predict or prevent spillover events. This work provides a foundation for transdisciplinary investigation of spillover and synthetic theory on zoonotic transmission.

The phenomenon of cross-species spillover is the defining characteristic of pathogens that transmit from vertebrate animals to humans (zoonoses). The public health burden that is presented by zoonoses includes outbreaks of pathogens such as Ebola virus, influenza A virus (H1N1) pdm09 and Middle East respiratory syndrome coronavirus (MERS-CoV), as well as the ongoing transmission of endemic pathogens, such as Salmonella spp., Leptospira spp., Trypanosoma spp., Mycobacterium spp. and West Nile virus ${ }^{1-6}$.

Spillover transmission is promoted by successive processes that enable an animal pathogen to establish infection in a human. The probability of zoonotic spillover is determined by interactions among several factors, including disease dynamics in the reservoir host, pathogen exposure and the within-human factors that affect susceptibility to infections. These factors can be partitioned into three functional phases that describe all major routes of transmission (FIG. 1). In the first phase, the amount of pathogen available to the human host at a given point in space and time, known as the pathogen pressure, is determined by interactions among reservoir host distribution, pathogen prevalence and pathogen release from the reservoir host, followed by pathogen survival, development and dissemination outside of the reservoir hosts. Second, human and vector behaviour determine pathogen exposure; specifically, the likelihood, route and dose of exposure. Third, genetic, physiological and immunological attributes of the recipient human host, together with the dose and route of exposure, affect the probability and severity of infection.

Each phase presents multiple barriers to the flow of a pathogen from a reservoir host to a recipient host. Spillover requires the pathogen to pass every barrier and thus can only occur when gaps align in each successive barrier within an appropriate window in space and time (FIG. 2). Consequently, zoonotic spillover is a relatively rare event, and although humans are continually exposed to many potentially infectious pathogens that are derived from other species, most of these microorganisms cannot infect or cause disease in humans $\mathrm{s}^{7-10}$.

This Opinion article focuses on spillover transmission, strictly defined as the processes that enable a pathogen from a vertebrate animal to establish infection in a human.
Although many recent articles have examined the fields of zoonoses or emerging pathogens ${ }^{2,3,10-15}$, a synthetic mechanistic understanding of animal-to-human transmission is lacking ${ }^{14,16}$. Much attention has been dedicated to the characterization of emerging infections $\mathrm{s}^{3,11,12,15}$; for example, the high frequency of zoonoses among emerging infections ${ }^{3,12}$, their socio-economic, environmental and ecological drivers ${ }^{2,13,17,18}$, and their phylogenetic and geographical distribution ${ }^{3}$. Similarly, the phases of zoonotic emergence in the human population ${ }^{11,14,18}$, adaptation and compatibility of zoonoses in humans ${ }^{10,11,19}$, and approaches to modelling the transmission of zoonoses ${ }^{14,16}$, have also been addressed in the literature. However, a comprehensive understanding of the processes that enable a pathogen from a vertebrate animal to establish infection in a human, and how these processes are hierarchically, functionally and quantitatively linked, remains a fundamental deficit in research on zoonoses ${ }^{14,16}$. In this Opinion article, we present a mechanistic structure that integrates the determinants of spillover and the interactions among them (FIG. 1). However, we do not address broader determinants of pathogen emergence or factors that affect disease severity or onward transmission in humans.

Although many of the individual determinants of spillover are subjects of intensive study, each is usually addressed in isolation in a specialized discipline (FIG. 2). Accordingly, the better-characterized factors become the focus of public health interventions. For example, reservoir hosts or vectors are often targeted for control before the concatenation and relative influence of processes that lead to spillover are understood, which sometimes leads to inefficient or even counterproductive interventions ${ }^{20}$. In other cases, multiple mechanisms are aggregated in analyses that obscure the interactions or heterogeneities that drive risk. Although the aggregation of mechanisms may be appropriate at times, identifying discrete mechanisms and how they interact to drive spillover is essential to recognize the assumptions that are implicit in simpler models, and to clarify which processes must be modelled explicitly and 
which can be combined. For example, does assessment of the risk of acquiring a zoonotic infection require the measurement of the pathogen burden carried by individual reservoir hosts, or is it sufficient to estimate the cumulative abundance of a pathogen in the environment over time? This is a key question for pathogens such as Leptospira interrogans, Giardia spp., and Escherichia coli O157, and the answer may depend on modes of contact and dose-response relationships in humans (see below). Models that integrate data from experiments, the field and epidemiological studies, even if only partially parameterized, may be necessary to make such determinations.

We describe how pathogens overcome a series of barriers to pass from reservoir hosts to humans. Crucially, nonlinear interactions among the barriers create bottlenecks in the flow of a pathogen between species. Such bottlenecks provide opportunities for public health interventions that could lead to substantial reductions in the risk of spillover. Alternatively, changing environmental or social conditions can alleviate these bottlenecks, which can cause surges in spillover infections. Our framework provides the foundation for operational models that are required for quantitative evidence-based risk analysis, preparedness, surveillance and control.

\section{Barriers to spillover}

The probability of spillover is determined by the interactions among the barriers and the associated bottlenecks that might prevent cross-species transmission. Many of these interactions are nonlinear and dynamic in space and time.

Pathogen pressure. The series of processes that culminate in pathogen pressure (the amount of a pathogen that is available to humans at a given point in time and space) includes pathogen dynamics in reservoir hosts, pathogen release from reservoir hosts, and pathogen survival or dispersal outside of reservoir hosts.

Pathogen dynamics in reservoir hosts can be represented as three variables that determine the distribution and intensity of infection in time and space: the density of reservoir hosts, the prevalence of infection among reservoir hosts, and the average intensity of infection in an infected reservoir host in time and space (Supplementary information S1 (box)). Many ecological and physiological factors influence these variables in communities of reservoir animals; however, two sets of factors are dominant.

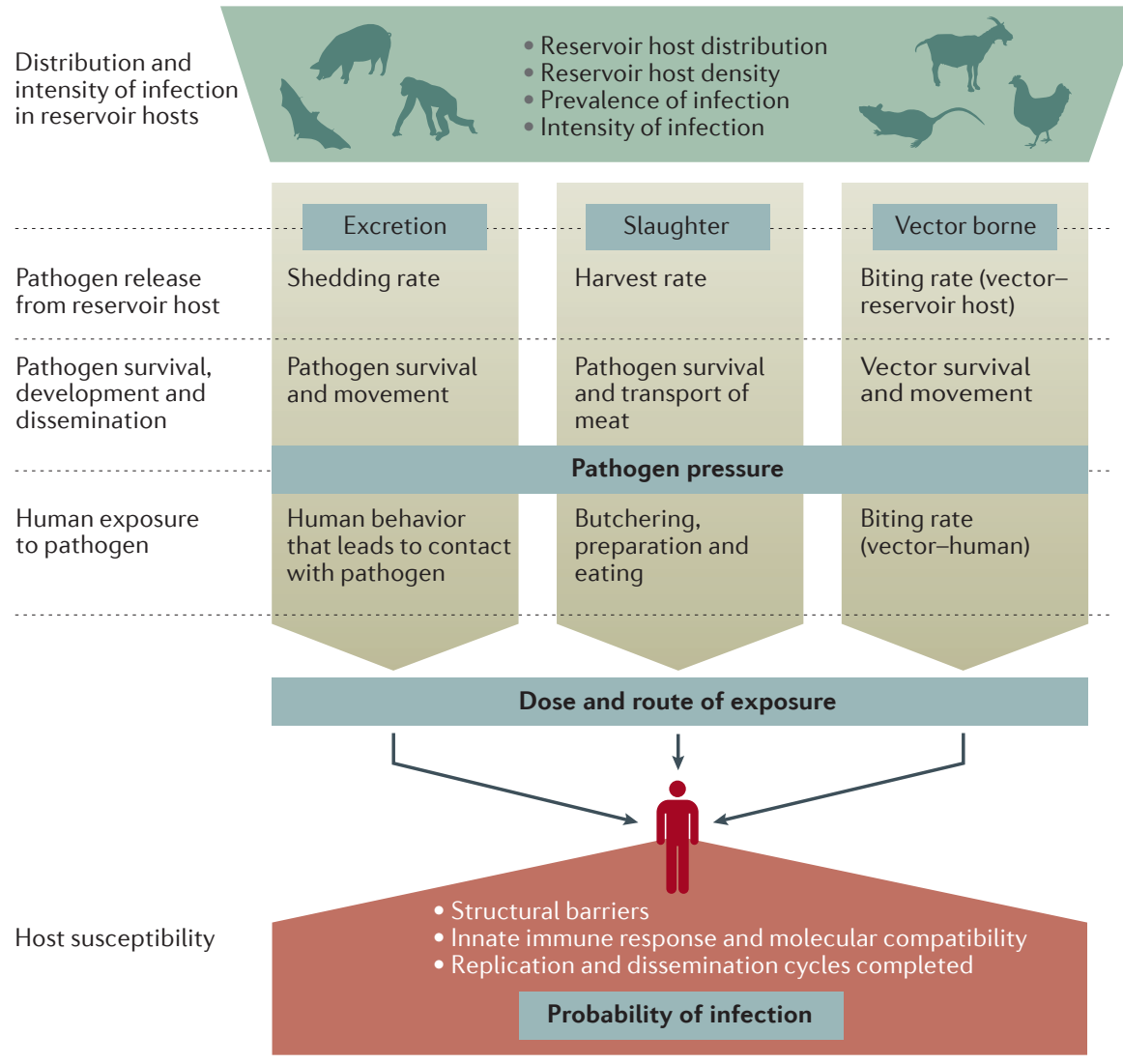

Figure 1 | Pathways to spillover. The risk of spillover is determined by a series of processes that link the ecological dynamics of infection in reservoir hosts, the microbiological and vector determinants of survival and dissemination outside of reservoir hosts, the epidemiological and behavioural determinants of exposure, and the within-host biological factors that shape the susceptibility of recipient hosts. The distribution and intensity of infection in reservoir hosts, followed by pathogen release, movement, survival and possible development to infectious stage, determine the pathogen pressure, which is defined as the amount of pathogen available to the recipient host at a given point in space and time. Pathogen pressure then interacts with the behaviour of the recipient host (and vector for vector-borne pathogens) to determine the likelihood, dose and route of exposure. A series of withinhost barriers then determine host susceptibility, and, therefore, the probability and severity of infection for a given pathogen dose.

The first set is the natural history of infection in hosts, which includes the duration, intensity and severity of infection and the level of shedding. Second, the movement and behaviour of hosts affect contact and the likelihood of exposure within and between species. These factors interact with the abundance, density, demographic turnover, spatial distribution and physiological state of hosts to determine the efficiency of spread ${ }^{21}$. Collectively, these processes determine how the pathogen is distributed across reservoir host populations. Such pathogen distribution can be highly variable (for example, pulses of Sin Nombre virus infections in deer mice (Peromyscus maniculatus) populations in response to climate-driven increases in population density) ${ }^{22}$, or stable (as illustrated by Mycobacterium bovis infections in populations of livestock and wildlife $)^{23}$.
The mode of pathogen release from reservoir hosts determines the major routes of transmission. Pathogens may be released in host excretions, through slaughter or through an arthropod vector (FIG. 1). The probability of a pathogen being released from a reservoir host is affected by its presence and viability in relevant tissues, such as the blood for many vector-borne pathogens, tissues contacted or consumed during butchering and eating for some food-borne pathogens, and tissues through which external shedding occurs for direct or environmental routes. For example, the viral load and excretion rates in the salivary glands are key determinants for the transmission of rabies virus from carnivores, whereas viral loads in the intestinal and respiratory tracts affect the transmission of avian influenza virus from poultry ${ }^{24-26}$. Likewise, the release of pathogenic Leptospira 
spp. from animal hosts requires colonization of the renal tubules ${ }^{27}$. The excreted pathogen load depends on the quantity of leptospires that effectively colonize the tubules ${ }^{28}$, the rate of release and the urinary output of the host $^{29}$. Moreover, the pathogen undergoes several changes in its lipopolysaccharide content and proteome during colonization and shedding in the urine $\mathrm{e}^{30,31}$, which suggests that priming in the renal milieu is required to adapt for survival and infectivity in the external environment. The rate of pathogen release is a crucial determinant of spillover risk, and care must be taken to appropriately formulate models that represent the rate of release for each route of transmission (BOX 1; Supplementary information S1 (box)).
Following the release of a pathogen from its reservoir host, the opportunity for spillover transmission is influenced by the duration of pathogen survival outside of its host, the extent of spatial dispersal through passive transport (for example, through water, on fomites or in the air), and possible pathogen reproduction or obligate developmental stages outside of the primary host (for example, Yersinia pestis, the causative agent of plague, must multiply within flea vectors before it can be transmitted to humans ${ }^{32}$ ). These processes can be represented as the probability that the pathogen (shed, harvested or colonized in a vector) survives and is infectious at a given point in time, and is dispersed or transported to a particular location (Supplementary information S1 (box)). Spillover of pathogens that have short survival times (for example, influenza A virus when transmitted through the respiratory route $)^{33,34}$ may require close interactions between reservoir and recipient hosts. Consequently, spillover patterns in recipient hosts correspond to the prevalence patterns in reservoir hosts. By contrast, if pathogens survive for sufficient periods of time outside of their reservoir hosts, they may be dispersed beyond the home range of the host through fomites or environmental transport. In this case, the release of a pathogen from its reservoir host and human exposure to the pathogen may become disconnected in space and time. An example is the spread of aerosolized Coxiella burnetii

a

Disease ecology, animal epidemiology, infectious disease dynamics, immunology, microbiology, veterinary medicine b

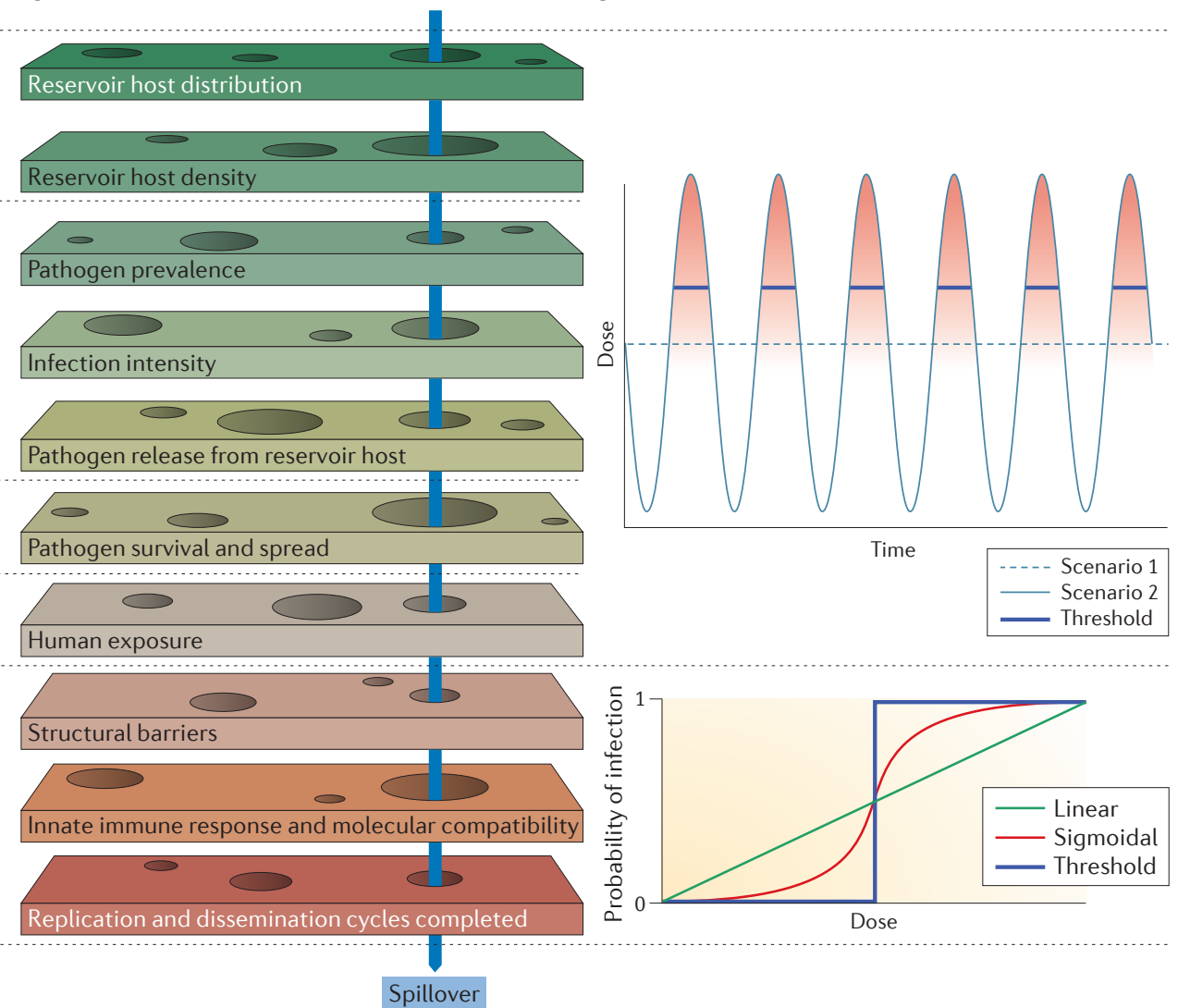

Figure 2 | Barriers to spillover and dose-response relationships. a|Determinants of spillover are being studied by researchers in many disciplines. $\mathbf{b} \mid$ A pathogen must overcome a series of barriers to transmit from one species to another. If any of these barriers is impenetrable, spillover cannot occur. Spillover of some pathogens requires that gaps (depicted as holes) in all of the barriers align within a narrow window in space and time (indicated by the blue arrow, see Supplementary information S2 (movie)). For other pathogens, protracted survival in the environment (for example, Bacillus anthracis spores ${ }^{109}$ ), or wide dissemination (for example, the spread of aerosolized Coxiella burnetii by wind ${ }^{35}$ ), may stagger the alignment of barriers to spillover. c| Top panel: hypothetical dose available over time for a given pathogen. In scenario 1 (dashed light blue line), the pathogen is excreted consistently from infected reservoir hosts. In scenario 2 (solid light blue line), the pathogen is excreted in regular but short high-intensity pulses over time. In both scenarios, the mean dose over the time interval is the same. Bottom panel: the likelihood that this dose will translate into infection depends on the functional form of the dose-response relationship. If the dose-response relationship is linear (green line), these two excretion scenarios generate the same total probability of spillover over the time interval shown. However, for nonlinear dose-response relationships, the total probability of spillover differs between scenarios. If the relationship is sigmoidal (red line), there is some probability of spillover whenever the dose exceeds zero (indicated by the intensity of the red shading in the top panel), but the total spillover probability in scenario 2 is markedly higher. In the extreme case in which the recipient host can be infected only by a dose that exceeds a sharp threshold, as suspected for Bacillus anthracis ${ }^{67,68,79}$, the pathogen in scenario 2 will spill over when the dose peaks above the threshold (blue solid line near peak), but the pathogen in scenario 1 will never spill over. 
by wind, which can lead to outbreaks of Q fever in humans that live several kilometres from the livestock reservoir hosts ${ }^{35}$.

As illustrated by rabies virus, pathogenic Leptospira spp. and E. coli O157 (FIG. 3), the bottlenecks that hinder the transfer of pathogens between species depend on the ecology of the reservoir host and the pathogen, and the interactions among the determinants of spillover. For example, the primary driver of pathogen pressure for rabies virus is the prevalence of infection in key hosts (such as domestic $\left.\operatorname{dogs}^{36}\right)$. Nonlinearities in rabies transmission generate a threshold effect in susceptible host density below which the pathogen cannot persist. These thresholds can be used to set vaccination targets for disease elimination ${ }^{37}$. By contrast, pathogen pressure of L. interrogans is also affected by fluctuations in reservoir host density (such as rodents ${ }^{29}$ ), and prevalence and shedding from infected animals ${ }^{29}$. However, if human exposure occurs through mechanisms that aggregate and disperse pathogens shed by many individuals (through accumulation in the environment, sustained survival after exiting the host ${ }^{38}$, and dispersal through rain, rivers and flood waters ${ }^{39}$ ), the detailed dynamics in reservoir hosts do not matter because they get integrated out by the environmental reservoir. In this scenario, spillover risk is determined by the aggregate pathogen pressure, human behaviours that determine exposure and the integrity of within-human barriers to infection. For example, when flooding mobilizes Leptospira spp. during the wet season in Brazil, human exposures can become widespread and epidemics of spillover infection can occur ${ }^{40}$. During these extreme environmental events, control efforts must focus on preventing exposure to contaminated sources (for example, by wearing protective clothing and boots ${ }^{41}$ ) and reducing the infectious inoculum rather than reducing the source of pathogen shedding, as the release of Leptospira spp. into the environment by animal reservoirs occurs before the extreme precipitation. Similarly, pathogen pressure of E. coli O157 is affected by the density of its cattle host population ${ }^{42}$, by variation in shedding among individuals and by prevalence in herds $^{43}$. Each of these factors can be highly skewed and seasonal ${ }^{44,45}$. If spillover events are driven by contact between humans and cattle, then variation in pathogen load among animals would interact with nonlinear doseresponse functions to determine spillover risk (see below). However, this individual variation matters less if human exposure

\section{Box 1 | The mathematics of spillover}

The opportunities for cross-species transmission are influenced by processes that occur at scales from molecules to landscapes (FIG. 1). These processes are subjects of intense study, and their characterization is complicated by their variability in space and time, nonlinear responses and interactions with outside factors. Consequently, it is impossible to integrate all of the determinants of spillover transmission - or to assess the effects of gaps in our knowledge about these determinants - without appropriate tools, such as mathematical and computational models ${ }^{107}$. In Supplementary information S1 (box), we present a general mathematical model of the spillover process, which provides a template for integrating our knowledge of processes for specific disease systems. This model framework essentially translates FIC. 1 into mathematical expressions. It allows for variation in space and time, and uses different formulations for transmission through pathogen excretion, slaughter or arthropod vectors.

The mathematical model reflects the modular nature of the spillover process, as emphasized in the main text, while highlighting dependencies among factors in ways such as the following:

- Factors that are linked to disease ecology of the reservoir host and the mode of pathogen release determine the amount of pathogen released to the environment or vector.

- Pathogen survival and transport outside of the animal host, which give rise to pathogen pressure at a particular place and time, are modelled with simple probability kernels.

- Human risk behaviours determine how this pathogen pressure translates to exposure dose.

- The probability of infection for a given dose and route of exposure is encapsulated in the dose-response relationship (FIG. 2C).

Mathematically, the focal point of this process is the dose to which the recipient host is exposed. All upstream factors come together, with appropriate functional dependencies, to shape this dose. To a reasonable approximation, which is consistent with current practice in quantitative microbial risk assessment ${ }^{108}$, the consequent risk of infection can be modelled independently through the dose-response relationship.

occurs after human-mediated dispersal of the pathogen through irrigation, meat processing and food transportation ${ }^{46-48}$. In this instance, outbreaks of $E$. coli $\mathrm{O} 157$ are determined by the pathogen pressure on vegetables or in hamburger meat, potentially derived from many sources. As the dose that is required for E. coli $\mathrm{O} 157$ spillover is thought to be very low ${ }^{49,50}$, public health policies aim to completely eliminate pathogen pressure in food that is processed for human consumption ${ }^{50}$. To achieve this goal, interventions are focused on creating successive bottlenecks in several barriers to spillover, including decreasing cattle density, preventing faecal contamination during meat processing and increasing cooking temperatures to reduce exposure dose in ground beef ${ }^{43,47,51}$. Cumulatively, these efforts are usually successful, but high levels of shedding from cattle during summer can sometimes overwhelm interventions $s^{47}$.

Exposure. The next phase of spillover exposure - bridges the upstream processes that generate pathogen pressure and the within-host processes in the recipient that determine whether a given dose generates a spillover infection (see below). The interaction between recipient hosts and pathogen pressure determines both the dose and the route of exposure. Different behaviours of the recipient host are relevant to exposure through different routes of transmission $^{52}$. Human behaviours, such as occupational interactions with reservoir host animals, the consumption of certain animal products or the use of particular environments, may increase the risk of infection ${ }^{53}$.

Exposure is often conceptualized as a simple point of contact. However, nonlinear interactions between pathogen pressure, human risk behaviour and environmental factors can lead to unexpected complexity, especially for vector-borne diseases. For example, in rats, both a high prevalence of $Y$. pestis and high mortality may be necessary to drive outbreaks of bubonic plague in humans. Widespread exposure of humans through flea bites occurs only after a decrease in the abundance of rats, which are the primary hosts of $Y$. pestis in peridomestic settings ${ }^{54}$. Indeed, historically, high rat mortality ('rat-fall') was an indication of an imminent human plague epidemic $^{32}$. Thus, killing rodents in response to cases of bubonic plague in humans could inadvertently increase the severity of the epidemic ${ }^{54}$. Conversely, and controversially, zooprophylaxis, which involves diverting vector bites from humans by increasing the local population density of another animal host, may decrease the risk of human exposure $^{55}$. For example, the presence of chickens and dogs in rural areas of Argentina 
decreased the rate at which Triatoma infestans transmitted Trypanosoma cruzi, the causative agent of Chagas disease, to humans ${ }^{56}$.

However, increasing the population density of reservoir hosts may also affect vector survival, vector abundance and pathogen prevalence in reservoir hosts, which, in turn, increases pathogen pressure and offsets reductions in human-vector contact rates ${ }^{56,57}$. These complexities highlight the need to understand the mechanisms that contribute to particular routes of spillover.

All of the factors that precede human exposure, mediated by human behaviour and environmental factors (FIG. 1), cumulate in the dose to which a host is exposed at a given location and time (the integral of the pathogen pressure in space and time to which the host has been exposed (Supplementary information S1 (box)).

\section{Probability of infection. Following} cross-species exposure of a recipient host, the within-host barriers and their interactions with the strain of pathogen determine the functional relationship between the pathogen dose and the likelihood that an infection will establish. Within-host barriers to infection vary widely and depend on the specific combinations of pathogen, host species and individual receptivity ${ }^{58}$. Physical barriers include the skin, mucous membranes, mucus, stomach acid or the absence of functional receptors that enable the pathogen to enter its target cells or tissues ${ }^{10}$. Interferoninduced and other innate immune responses may be triggered after the initial infection of a cell, resulting in protective mechanisms such as apoptosis or the induction of interferon-induced resistance in surrounding cells $^{59}$. In addition, interfering defensive proteins in the host cell cytoplasm may block the replication of intracellular pathogens. In other cases, cells lack functional host factors that are required for the replication of the

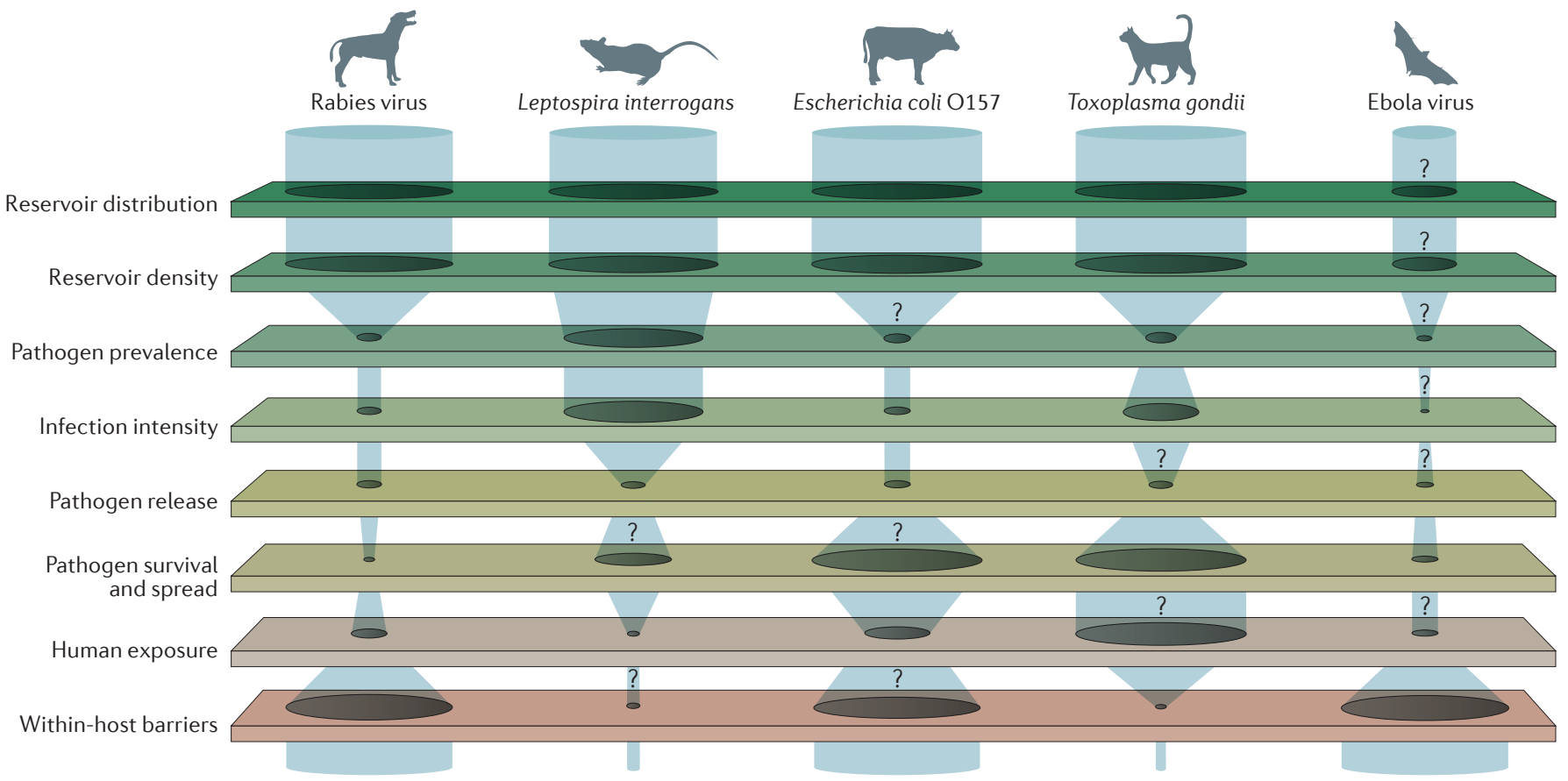

Figure 3 | Bottlenecks to spillover. Different barriers permit or constrain the flow of pathogens from one species to another. The figure is illustrative, owing to the lack of sufficient data for more than one or two barriers for any given system. The width of the gaps in barriers represents the ease with which a pathogen can flow through the barriers and will vary depending on context. The question marks represent points at which the barriers are especially poorly understood and highlight gaps in our knowledge of some pathogens that are of global concern (for example, the lack of information on disease dynamics in reservoir hosts of Ebola virus). Many rabies virus reservoirs, such as domestic dogs, are widely distributed. The prevalence of rabies virus is generally low and the incidence of spillover closely tracks the prevalence of infection in the reservoir host. Rabies virus is almost always fatal to spillover hosts ${ }^{25}$. Interventions are usually aimed at reducing the prevalence in reservoir hosts through vaccination ${ }^{37}$. Leptospira interrogans survives in water and soil after being shed in the urine of a wide range of rodents and other reservoir hosts ${ }^{29}$. Key bottlenecks to the zoonotic spillover of this pathogen are exposure and within-host barriers. For example, during floods in Brazil, many humans that are exposed do not become infected, probably because the initial within-host barrier, the skin, is not penetrated ${ }^{41}$. However, once L. interrogans penetrates the skin (for example, through skin wounds), 1-10 leptospires may be sufficient to cause systemic infection ${ }^{110}$. Therefore, wearing protective clothing and boots is an effective control measure ${ }^{41}$. Important bottlenecks to Escherichia coli 0157 spillover include heterogeneous shedding from cattle ${ }^{43,44}$ (although it is still unknown whether super-shedding is a characteristic of particular individuals or is a transient phase that occurs in most cattle ${ }^{42}$ ). In some contexts, exposure is an important bottleneck; for example, when the pathogen is eliminated from food through cooking. Widespread dispersal leads to uncertainties about the source of many outbreaks ${ }^{46,47}$, and weak within-human barriers enable low doses of E. coli to cause infection ${ }^{49,50}$. Humans are frequently exposed to Toxoplasma gondii carried by domestic cats and intermediate hosts, but the parasite rarely causes disease because most humans have strong within-host immunological barriers. Cats are widely and densely distributed, but the prevalence of T. gondii is low and cats shed oocysts only once in their lifetime ${ }^{111}$. However, sporulated oocysts survive in the environment for long periods of time ${ }^{112}$. Limiting exposure to oocysts may prevent spillover; however, this is challenging when it is unclear whether cats or the environment are the major sources of infection in humans ${ }^{111,113}$. Ebola virus has not been isolated from bats and the definitive reservoir bat species is unknown ${ }^{114}$; therefore, characteristics of infection in bats are unknown $n^{114,115}$. The pathogen is released through excretion or slaughter, then survives for up to a week, depending on the environmental conditions ${ }^{116}$. The most tractable bottlenecks for intervention may be the zoonotic exposure of humans through interaction with bats, bushmeat or the carcasses of other species ${ }^{97,117,118}$, because once exposed, the within-host barriers to Ebola virus may be extremely low ${ }^{119}$. 
pathogen ${ }^{60,61}$. Even when pathogens can replicate within cells, several barriers can prevent their transmission to other cells $^{62,63}$ and thus the establishment of an infection. For example, avian influenza virus must pass through a series of within-host barriers to infect a human, including mucins in respiratory tract excretions, specific receptor molecules that constrain virus entry into cells and have different distributions in the respiratory tracts of different host species, suboptimal viral polymerase that restricts the ability of the virus to replicate in cells of the human respiratory tract, viral neuraminidase that is inefficient in its role in the release of influenza viruses from infected cells, and innate immune responses that are initiated early and that block infection in both infected and neighbouring cells ${ }^{63,64}$.

From an epidemiological perspective, these within-host interactions between zoonotic pathogens and hosts can be encapsulated by the functional relationship between pathogen dose and the probability of an infection. Although there is much to learn about dose-response relationships, they are expected to be nonlinear as, at minimum, they must saturate at high doses because the probability of infection cannot exceed one $e^{65}$. This nonlinearity imposes a filter on the dynamics of pathogen pressure and exposure (FIG. 2c). If the dose-response relationship is highly nonlinear, such that small changes in dose lead to large changes in the probability of an infection, then variation in any of the upstream factors that culminate in an exposure dose (including released dose, pathogen survival and human behaviour) may have disproportionate effects on the probability of spillover. Such effects could generate opportunities for targeted control measures. Moreover, nonlinear dose-response relationships may imply that infrequent high-intensity exposures are more likely to cause spillover infections than continuous low-intensity excretion. This phenomenon has been reported for occupational exposure to Bacillus anthracis aerosols; tannery workers who were exposed to infrequent high doses of $B$. anthracis spores in imported goat hair were more likely to die of anthrax than those who were exposed to frequent low doses of B. anthracis spores $^{66-68}$. Conversely, if doses are far below the inflection point on the dose-response curve (FIG. 2c), then the system may be insensitive to changes in dose. If the doseresponse function is close to linear, the total exposure dose over time is equal and host responses do not change as a consequence of early exposures, then longer-term exposure to a low but constant dose may generate the same probability of infection as intermittent high-intensity exposures (FIG. 2C).

The genetic, immunological and physiological state of the host also can modulate the dose-response relationship. Immunosuppression (for example, due to AIDS, immunosuppressive drugs, co-infections or malnutrition) increases gaps in within-host barriers, which shifts dose-response curves and increases susceptibility ${ }^{69,70}$. For example, in immunosuppressed hosts, the decreased number or activity of lymphocytes can reduce the dose that is required to establish an infection with the widespread pathogen Toxoplasma gondii, or cause the loss of control of $T$. gondii infections that are usually kept in check by sustained immune pressure ${ }^{71}$ (FIG. 3). Seasonality in human immune function (for example, enhanced baseline inflammation and altered cellular composition of the immune system in winter compared with summer) may also alter the permeability of within-host barriers by altering the magnitude and speed of immune responses ${ }^{72}$. Finally, the probability and severity of infection at a given dose are shaped by host genetics ${ }^{73}$; triathletes with a particular gene polymorphism were at increased risk of leptospirosis after swallowing lake water compared with athletes who lacked this polymorphism ${ }^{74}$.

Many of the interactions at the crossroads of exposure, inoculum dose and host response are poorly understood. Therefore, very little is known about the interactions between dose, timing of exposure and probability of infection. The current dose-response paradigm is based on discrete transient exposures, but the effects of protracted or cumulative exposure to environmental pathogens (for example, to low concentrations of Leptospira spp. in floodwater) are unclear $^{75}$. Repeated low-dose exposure can increase host immunity to infection (for example, as postulated for poultry handlers who are exposed to avian influenza ${ }^{76}$, dairy farmers who are exposed to E. coli O157 (REF. 77) and mice that are exposed to continuous infections of parasites $\left.^{78}\right)$. However, increases in immunity are not always observed; for example, such effects on immunity were not observed in tannery workers who were exposed to B. anthracis ${ }^{67,68,79}$. Moreover, it may be difficult to differentiate between a cumulative dose effect and the increasing opportunity to initiate an infection with each additional low-dose exposure (if each infectious unit has a probability of causing an infection that is above zero $)^{20,80}$.
Once a pathogen has penetrated the within-host barriers to replicate and disseminate in the new host, the outcome of the infection may range from subclinical elimination of the microorganism to the death of the new host, and from dead-end spillover infection to sustained human-to-human transmission. For many important zoonotic pathogens, such as HIV or Zika virus, the transmission that drives the current public health crisis is human-to-human ${ }^{81,82}$ and the events that led to spillover are long past. Although understanding disease severity and onward transmission is essential for understanding the consequences of emerging infectious diseases, these processes are beyond the scope of this article. Our current knowledge of the biological features of pathogens and characteristics of host-pathogen interactions that determine these outcomes are described elsewhere (for example, see REFS 83,84).

\section{Assessing zoonotic risk}

When gaps in barriers to spillover are highly dynamic in time and space, they may vary asynchronously, so that the alignment of gaps in all barriers may be fleeting and spillover may seem random (Supplementary information S2 (movie)). Research methods that group multiple barriers or integrate data over space and time may not capture these dynamics. For example, ecological niche models are often used to study zoonotic risk by assessing the distribution of reservoir hosts or vectors ${ }^{85}$, but this approach overlooks variation in downstream barriers that might drive risk. Alternatively, niche models that are based on the documented occurrence of spillover may capture the accumulated distribution of all conditions that enabled barriers to be breached over time (FIG. 1), but they cannot isolate the precise barriers that affect spillover risk (for example, see REF. 86). Therefore, niche models tend to overestimate the spatial range of spillover risk and do not readily enable extrapolation to novel conditions ${ }^{87}$. Examples of this include Hendra virus and Marburg virus, which can be excreted in discrete temporal and spatial pulses from their bat reservoir hosts ${ }^{20,88,89}$. However, for spillover, shedding must align with environmental and bat population conditions that generate levels of pathogen pressure that are sufficient to produce an infectious dose (FIG. 2), and with exposure behaviours and susceptibility of the recipient hosts. As some of these conditions vary among seasons and years, the pattern of outbreaks in livestock or humans has high spatial and temporal variability ${ }^{20,89}$. However, 
as niche models often summarize risk across large areas and long durations, they overlook important heterogeneities and they lack the specificity that is required for public health intervention. Although niche models can help to identify regional-to-continental concentrations of risk ${ }^{90,91}$, risk assessments that are more quantitative and more precise with regard to space, time and which barriers they address are needed to guide concrete action.

Epidemiological investigations of spillover also need to account for conditions that are highly dynamic in space and time. If the alignment of gaps in all barriers is fleeting, delayed diagnoses or inconsistent case detection may delay outbreak investigations until the conditions that enabled spillover have changed. Similarly, investigations are sometimes triggered once the case count becomes high. These challenges differ among pathogens with different values of $\mathrm{R}_{0}$ (the basic reproductive number or expected number of secondary infections caused by a typical infected individual in a susceptible population). For supercritical pathogens with $\mathrm{R}_{0}>1$, which can cause major epidemics through sustained transmission in human populations (for example, Ebola virus, Zika virus and the pandemic strain of severe acute respiratory syndrome coronavirus (SARS$\mathrm{CoV})^{4,81,92}$ ), spillover becomes challenging to study because a given human case is likely to be far removed in time or space from the spillover event that triggered an outbreak. Subcritical pathogens with $0<\mathrm{R}_{0}<1$, which cause self-limiting outbreaks or 'stuttering chains' in human populations (for example, monkeypox or avian influenza viruses ${ }^{93,94}$ ), raise distinct challenges because any given individual could have been infected by either an animal or a human source ${ }^{16}$. It is easiest to study the spillover of pathogens with $\mathrm{R}_{0}=0$ that are not transmitted between humans (for example, rabies virus or West Nile virus $^{25,95}$ ), in which every case is an instance of spillover. The 2014-2015 Ebola virus epidemic in West Africa is a prime example whereby delayed response and investigation prevented researchers from reconstructing the conditions that initiated the human epidemic of a supercritical pathogen ${ }^{96,97}$. Ebola virus infection is an extreme example of spillover infection that only occurs during the rare alignment of gaps in barriers, and, accordingly, the precise determinants of risk are poorly understood (FIG. 3). By contrast, for other zoonoses, such as trypanosomiasis in some parts of Africa, incidence is high because the pathogen flows through consistently wide gaps in barriers to infection (for example, common exposure to infected animal hosts and tsetse fly vectors, and low resistance in humans due to the ability of trypanosomes to neutralize or avoid human innate immune activity ${ }^{98,99}$ ). In all scenarios, irrespective of the frequency with which gaps align, the concept of hierarchical barriers can be used to organize and quantify the conditions that enable spillover.

The influence of particular barriers may vary in space and time, and this variation - coupled with data on realized spillover events - can help elucidate factors that shape infection risk, even in the absence of information on other barriers. In the westernmost province of the Democratic Republic of Congo, the observed lack of monkeypox spillover, despite high seroprevalence in the suspected reservoir hosts (Heliosciurus spp. and Funisciuris spp.), was attributed to cultural norms that forbade the consumption of small rodents ${ }^{100}$. The inconsistency between ecological data that suggested high pathogen pressure and epidemiological data that indicated a lack of spillover, focused attention on human behaviours that affect the probability of exposure. Research approaches that integrate data on multiple barriers are more likely to discern such behavioural effects.

Broad-scale discovery of novel microorganisms has the potential to characterize the pool of possible zoonotic pathogens and provide valuable baseline information ${ }^{101,102}$. However, each of the $\sim 63,000$ species of mammals, birds, reptiles, amphibians and fish ${ }^{103}$ contains a multitude of infectious viruses, bacteria and parasites ${ }^{101,102,104-106}$. Although each of these microorganisms and parasites can be viewed as a potential pathogen, the vast majority may not cause disease in their natural hosts, and the extent to which they infect or cause pathology in other species, including humans, is unknown ${ }^{7,9,10}$. Therefore, discovery alone cannot address the potential risk of spillover. The translation of new discoveries of microorganisms into guidance for public health practitioners requires the identification of the barriers to microbial infection of humans, the conditions that facilitate the breaching of these barriers, and, therefore, the microbiological and environmental contexts that pose the greatest risk to human populations. For the foreseeable future, the greatest practical contribution of pathogen discovery and sequence characterization to the epidemiology of emerging pathogens is likely to be in the rapid post hoc identification of novel pathogens after spillover.

\section{Outlook}

The framework presented in this Opinion article highlights that an important frontier in research on zoonotic spillover is to understand the functional and quantitative links among the determinants of spillover. To our knowledge, all of the processes that are necessary to achieve spillover have not been connected, compared and quantified for any single zoonotic pathogen. We address this gap, in part, by introducing a conceptual and quantitative model that can be used to integrate existing data, identify high-priority data gaps, investigate conditions that widen or align gaps in barriers to spillover, and identify the best gaps on which to focus intervention efforts. We suggest that future research focuses on developing case studies that contribute to fully quantifying the determinants of spillover and their linkages, with the goal of making operational contributions to risk assessment. We provide a mathematical framework that formalizes the ideas presented here to guide the formulation of mechanistic spillover models for particular zoonotic pathogens (BOX 1; Supplementary information S1 (box)). We anticipate that this synthetic framework will provide a foundation for cross-scale data integration, transdisciplinary investigation, and a new body of theory on spillover that is necessary for risk assessment and public health planning.

Raina K. Plowright is at the Department of Microbiology and Immunology, Montana State University, Bozeman, Montana 59717, USA.

Colin R. Parrish is at the Baker Institute for Animal Health, College of Veterinary Medicine, Cornell University, Ithaca, New York 14853, USA.

Hamish McCallum is at the Griffith School of Environment, Griffith University, Brisbane, Queensland 4111, Australia.

Peter J. Hudson is at the Center for Infectious Disease Dynamics, Pennsylvania State University, State College, Pennsylvania 16802, USA.

Albert I. Ko is at the Department of Epidemiology of Microbial Diseases, Yale School of Public Health, New Haven, Connecticut 06520-8034, USA.

Andrea L. Graham is at the Department of Ecology \& Evolutionary Biology, Princeton University, Princeton, New Jersey 08544, USA.

James O. Lloyd-Smith is at the Department of Ecology \& Evolutionary Biology, University of California, Los Angeles, Los Angeles, California 90095-7239, USA; and at Fogarty International Center, National Institutes of Health, Bethesda, Maryland 20892-2220, USA.

Correspondence to R.K.P. raina.plowright@montana.edu doi: $10.1038 /$ nrmicro.2017.45 Published online 30 May 2017 
1. Christou, L. The global burden of bacterial and viral zoonotic infections. Clin. Microbiol. Infect. 17, 326-330 (2011)

2. Morens, D. M., Folkers, G. K. \& Fauci, A. S. The challenge of emerging and re-emerging infectious diseases. Nature 430, 242-249 (2004).

3. Jones, K. E. et al. Global trends in emerging infectious diseases. Nature 451, 990-993 (2008).

This study analyses the general phylogenetic and geographical risk factors for many different emerging diseases, as well as temporal and spatial trends in emerging infections.

4. Briand, S. et al. The international Ebola emergency. N. Engl. J. Med. 371, 1180-1183 (2014).

5. Smith, G. J. et al. Origins and evolutionary genomics of the 2009 swine-origin H1N1 influenza A epidemic. Nature 459, 1122-1125 (2009)

6. Fevre, E. M., Wissmann, B. V., Welburn, S. C. $\&$ Lutumba, P. The burden of human African trypanosomiasis. PLoS Negl. Trop. Dis. 2, e333 (2008).

7. Grice, E. A. \& Segre, J. A. The skin microbiome Nat. Rev. Microbiol. 9, 244-253 (2011).

8. Guarner, F. \& Malagelada, J.-R. Gut flora in health and disease. Lancet 361, 512-519 (2003).

9. Gilbert, S. F., Sapp, J. \& Tauber, A. I. A symbiotic view of life: we have never been individuals. Q. Rev. Biol. 87, 325-341 (2012)

10. Parrish, C. R. et al. Cross-species virus transmission and the emergence of new epidemic diseases. Microbiol. Mol. Biol. Rev. 72, 457-470 (2008). This article reviews the general features that are associated with the emergence of viruses in new hosts to cause epidemics or pandemics.

11. Woolhouse, M. E. \& Gowtage-Sequeria, S. Host range and emerging and reemerging pathogens. Emerg. Infect. Dis. 11, 1842-1847 (2005).

12. Taylor, L. H., Latham, S. M. \& Woolhouse, M. E. J. Risk factors for human disease emergence. Phil. Trans. R. Soc. Lond. B Biol. Sci. 356, 983-989 (2001).

13. Morse, S. S. Factors in the emergence of infectious diseases. Emerg. Infect. Dis. 1, 7-15 (1995).

14. Lloyd-Smith, J. O. et al. Epidemic dynamics at the human-animal interface. Science 326, 1362-1367 (2009).

This study delineates stages of zoonoses on the basis of changes in transmissibility, as reflected in $\mathbf{R}_{0}$. It also reviews the literature on modelling transmission dynamics of zoonoses and identifies gaps in our knowledge.

15. Johnson, C. K. et al. Spillover and pandemic properties of zoonotic viruses with high host plasticity. Sci. Rep. 5, 14830 (2015).

16. Lloyd-Smith, J. O., Funk, S., McLean, A. R., Riley, S. $\&$ Wood, J. L. Nine challenges in modelling the emergence of novel pathogens. Epidemics 10, 35-39 (2015).

17. Gortazar, C. et al. Crossing the interspecies barrier: opening the door to zoonotic pathogens. PLoS Pathog. 10, e 1004129 (2014).

18. Wolfe, N. D., Dunavan, C. P. \& Diamond, J. Origins of major human infectious diseases. Nature $\mathbf{4 4 7}$ 279-283 (2007)

19. Pepin, K. M., Lass, S., Pulliam, J. R., Read, A. F. \& Lloyd-Smith, J. O. Identifying genetic markers of adaptation for surveillance of viral host jumps. Nat. Rev. Microbiol. 8, 802-813 (2010).

20. Plowright, R. K. et al. Ecological dynamics of emerging bat virus spillover. Proc. R. Soc. B Biol. Sci. 282, 20142124 (2015). This study outlines the conditions that enable spillover of bat viruses into other hosts and provides an example of the infections that are the subject of this review.

21. Hudson, P. J., Rizzoli, A. R. Grenfell, B. T. Heesterbeek, H. \& Dobson, A. P. The Ecology of Wildlife Diseases (Oxford Univ. Press, 2002)

22. Hjelle, B. \& Glass, G. E. Outbreak of hantavirus infection in the Four Corners region of the United States in the wake of the 1997-1998 El NinoSouthern Oscillation. J. Infect. Dis. 181, 1569-1573 (2000).

23. Thoen, C. O., Steele, J. H. \& Kaneene, J. B. Zoonotic Tuberculosis: Mycobacterium bovis and Other Pathogenic Mycobacteria (John Wiley \& Sons, 2014).

24. Ducatez, M., Webster, R. \& Webby, R. Animal influenza epidemiology. Vaccine 26, D67-D69 (2008)

25. Rupprecht, C. E., Hanlon, C. A. \& Hemachudha, T Rabies re-examined. Lancet Infect. Dis. 2, 327-343 (2002).
26. Webster, R. in Viral Zoonoses and Food of Animal Origin (eds Kaaden, O.-R., Czerny, C.-P. \& Eichhorn, W.) 105-113 (Springer, 1997)

27. Ko, A. I., Goarant, C. \& Picardeau, M. Leptospira: the dawn of the molecular genetics era for an emerging zoonotic pathogen. Nat. Rev. Microbiol. 7, 736-747 (2009).

28. Costa, F. et al. Influence of household rat infestation on Leptospira transmission in the urban slum environment. PLoS Negl. Trop. Dis. 8, e3338 (2014).

29. Costa, F. et al. Patterns in Leptospira shedding in Norway rats (Rattus norvegicus) from Brazilian slum communities at high risk of disease transmission. PLoS Negl. Trop. Dis. 9, e0003819 (2015).

30. Monahan, A. M., Callanan, J. J. \& Nally, J. E. Proteomic analysis of Leptospira interrogans shed in urine of chronically infected hosts. Infect. Immun. 76 4952-4958 (2008)

31. Nally, J. E., Chow, E., Fishbein, M. C., Blanco, D. R. \& Lovett, M. A. Changes in lipopolysaccharide O antigen distinguish acute versus chronic Leptospira interrogans infections. Infect. Immun. 73, 3251-3260 (2005)

32. Smego, R., Frean, J. \& Koornhof, H. Yersiniosis I: microbiological and clinicoepidemiological aspects of plague and non-plague Yersinia infections. Eur. J. Clin. Microbiol. Infect. Dis. 18, 1-15 (1999).

33. Weber, T. P. \& Stilianakis, N. I. Inactivation of influenza A viruses in the environment and modes of transmission: a critical review. J. Infect. 57, 361-373 (2008)

34. Koopmans, M. et al. Transmission of H7N7 avian influenza A virus to human beings during a large outbreak in commercial poultry farms in the Netherlands. Lancet 363, 587-593 (2004).

35. Tissot-Dupont, H., Amadei, M.-A., Nezri, M. $\&$ Raoult, D. Wind in November, Q fever in December. Emerg. Infect. Dis. 10, 1264 (2004).

36. Hampson, K. et al. Synchronous cycles of domestic dog rabies in sub-Saharan Africa and the impact of control efforts. Proc. Natl Acad. Sci. USA 104 7717-7722 (2007)

37. Brochier, B. et al. Large-scale eradication of rabies using recombinant vaccinia-rabies vaccine. Nature 354, 520-522 (1991).

38. Andre-Fontaine, G., Aviat, F. \& Thorin, C. Waterborne leptospirosis: survival and preservation of the virulence of pathogenic Leptospira spp. in fresh water. Curr. Microbiol. 71, 136-142 (2015)

39. Lau, C. L., Smythe, L. D., Craig, S. B. \& Weinstein, P. Climate change, flooding, urbanisation and leptospirosis: fuelling the fire? Trans. R. Soc. Trop. Med. Hyg. 104, 631-638 (2010)

40. Reis, R. B. et al. Impact of environment and social gradient on Leptospira infection in urban slums. PLoS Negl. Trop. Dis. 2, e228 (2008).

41. Phraisuwan, P. et al. Leptospirosis: skin wounds and control strategies, Thailand, 1999. Emerg. Infect. Dis. 8, 1455-1459 (2002).

42. Spencer, S. E., Besser, T. E., Cobbold, R. N. \& French, N. P. 'Super'or just 'above average'? Supershedders and the transmission of Escherichia coli O157: $\mathrm{H} 7$ among feedlot cattle. J. R. Soc. Interface 12, 0446 (2015)

This study examines the dynamics of $E$. coli transmission and the roles of super-shedder individuals in those processes.

43. Matthews, L. et al. Heterogeneous shedding of Escherichia coli $\mathrm{O} 157$ in cattle and its implications for control. Proc. Natl Acad. Sci. USA 103, 547-552 (2006).

44. Hancock, D., Besser, T., Rice, D., Herriott, D. ¿ Tarr, P. A longitudinal study of Escherichia coli O157 in fourteen cattle herds. Epidemiol. Infect. 118 193-195 (1997).

45. Besser, T. E., Davis, M. A. \& Walk, S. T. in Population Genetics of Bacteria: A Tribute to Thomas S. Whittam (eds Walk, S. T. \& Feng, P. C. H.) 303-324 (2011).

46. Gerba, C. P. \& Smith, J. E. Sources of pathogenic microorganisms and their fate during land application of wastes. J. Environ. Qual. 34, 42-48 (2005).

47. Elder, R. O. et al. Correlation of enterohemorrhagic Escherichia coli 0157 prevalence in feces, hides, and carcasses of beef cattle during processing. Proc. Natl Acad. Sci. USA 97, 2999-3003 (2000). This study calculates the decreasing pathogen pressure (availability for human exposure) of $E$. coli 0157 as carcasses progress through the various stages of processing at meat processing plants.

48. Pennington, H. Escherichia coli O157. Lancet 376 , 1428-1435 (2010)
49. Teunis, P., Ogden, I. \& Strachan, N. Hierarchical dose response of $E$. coli O157: $\mathrm{H} 7$ from human outbreaks incorporating heterogeneity in exposure. Epidemiol. Infect. 136, 761-770 (2008).

50. Tuttle, J et al. Lessons from a large outbreak of Escherichia coli O157:H7 infections: insights into the infectious dose and method of widespread contamination of hamburger patties. Epidemiol. Infect 122, 185-192 (1999).

51. Cobbold, R. N. et al. Rectoanal junction colonization of feedlot cattle by Escherichia coli O157: $\mathrm{H} 7$ and its association with supershedders and excretion dynamics. Appl. Environ. Microbiol. 73, 1563-1568 (2007).

52. Cascio, A., Bosilkovski, M., Rodriguez-Morales, A. \& Pappas, G. The socio-ecology of zoonotic infections. Clin. Microbiol. Infect. 17, 336-342 (2011).

53. Macpherson, C. N. Human behaviour and the epidemiology of parasitic zoonoses. Int. J. Parasitol. 35, 1319-1331 (2005)

54. Keeling, M. J. \& Gilligan, C. A. Metapopulation dynamics of bubonic plague. Nature 407, 903-906 (2000).

This study uses dynamic models to explain historical patterns of bubonic plague, and shows that, counterintuitively, culling rats may exacerbate plague.

55. Hess, A. \& Hayes, R. O. Relative potentials of domestic animals for zooprophylaxis against mosquito vectors of encephalitis. Am. J. Trop. Med. Hyg. 19, 327-334 (1970).

56. Gürtler, R. E. et al. Domestic animal hosts strongly influence human-feeding rates of the Chagas disease vector Triatoma infestans in Argentina. PLoS Negl. Trop. Dis. 8, e2894 (2014).

57. Kilpatrick, A. M. \& Randolph, S. E. Drivers, dynamics, and control of emerging vector-borne zoonotic diseases. Lancet 380, 1946-1955 (2012).

58. Schmid-Hempel, P. Variation in immune defence as a question of evolutionary ecology. Proc. Biol. Sci. 270 , 357-366 (2003)

59. Akira, S., Uematsu, S. \& Takeuchi, O. Pathogen recognition and innate immunity. Cell 124, 783-801 (2006).

60. Trobaugh, D. W. \& Klimstra, W. B. MicroRNA regulation of RNA virus replication and pathogenesis. Trends Mol. Med. 23, 80-93 (2017).

61. Duggal, N. K. \& Emerman, M. Evolutionary conflicts between viruses and restriction factors shape immunity. Nat. Rev. Immunol. 12, 687-695 (2012)

62. Air, G. M. \& Laver, W. G. The neuraminidase of influenza virus. Proteins 6, 341-356 (1989).

63. Kuiken, T. et al. Host species barriers to influenza virus infections. Science 312, 394-397 (2006).

64. Lipsitch, M. et al. Viral factors in influenza pandemic risk assessment. eLife 5, e18491 (2016).

65. Schmid-Hempel, P. \& Frank, S. A. Pathogenesis, virulence, and infective dose. PLoS Pathog. 3, e147 (2007).

66. Brachman, P. S. \& Fekety, F. R. Industrial anthrax Ann. NY Acad. Sci. 70, 574-584 (1958).

67. Brachman, P. S., Kaufman, A. \& Dalldorf, F. G. Industrial inhalation anthrax. Bacteriol. Rev. 30, 646 (1966).

This study is one of the only studies to calculate the risk of spillover infection using comparable doses administered over time and provides evidence for the outcome of repeated low-dose versus single high-dose exposure.

68. Coleman, M. E., Thran, B., Morse, S. S., HughJones, M. \& Massulik, S. Inhalation anthrax: dos response and risk analysis. Biosecur. Bioterror. 6 , 147-160 (2008)

69. Bollaerts, K et al. Human salmonellosis: estimation of dose-illness from outbreak data. Risk Anal. 28 , 427-440 (2008)

70. Kau, A. L., Ahern, P. P., Griffin, N. W., Goodman, A. L. $\Sigma$ Gordon, J. I. Human nutrition, the gut microbiome and the immune system. Nature 474, 327-336 (2011)

71. Greene, C. E. Infectious Diseases of the Dog and Cat (Elsevier Health Sciences, 2013).

72. Dopico, X. C. et al. Widespread seasonal gene expression reveals annual differences in human immunity and physiology. Nat. Commun. 6, 7000 (2015).

73. Gingles, N. A. et al. Role of genetic resistance in invasive pneumococcal infection: identification and study of susceptibility and resistance in inbred mouse strains. Infect. Immun. 69, 426-434 (2001) 
74. Lingappa, J et al. HLA-DO6 and ingestion of contaminated water: possible gene-environment interaction in an outbreak of leptospirosis Genes Immun. 5, 197-202 (2004).

75. Pujol, J. M., Eisenberg, J. E., Haas, C. N. \& Koopman, J. S. The effect of ongoing exposure dynamics in dose response relationships. PLoS Comput. Biol. 5, e1000399 (2009).

76. Yang, W. \& Shaman, J. Does exposure to poultry and wild fowl confer immunity to H5N1? Chin. Med. J. 127, 3335 (2014)

77. Reymond, D. et al. Neutralizing antibodies to Escherichia coli vero cytotoxin 1 and antibodies to O157 lipopolysaccharide in healthy farm family members and urban residents. J. Clin. Microbiol. 34 2053-2057 (1996)

78. Scott, M. High transmission rates restore expression of genetically determined susceptibility of mice to nematode infections. Parasitology 132, 669-679 (2006).

79. Cohen, M. L. \& Whalen, T. Implications of low level human exposure to respirable $B$. anthracis. Appl. Biosafety 12, 109 (2007).

80. French, N., Kelly, L., Jones, R. \& Clancy, D. Doseresponse relationships for foot and mouth disease in cattle and sheep. Epidemiol. Infect. 128, 325-332 (2002).

81. Faria, N. R. et al. Zika virus in the Americas: early epidemiological and genetic findings. Science 352 345-349 (2016).

82. Hahn, B. H. Shaw, G. M. De Cock, K. M. \& Sharp, P. M. AIDS as a zoonosis: scientific and public health implications. Science 287, 607-614 (2000)

83. Geoghegan, J. L., Senior, A. M., Di Giallonardo, F. \& Holmes, E. C. Virological factors that increas the transmissibility of emerging human viruses. Proc. Natl Acad. Sci. USA 113, 4170-4175 (2016) This study identifies and quantifies biological features of viruses that best determine human infection and transmissibility between humans.

84. Casadevall, A. \& Pirofski, L. Host-pathogen interactions: the attributes of virulence. J. Infect. Dis. 184, 337-344 (2001).

85. Miller, R. H. et al. Ecological niche modeling to estimate the distribution of Japanese encephalitis virus in Asia. PLoS Negl. Trop. Dis. 6, e 1678 (2012).

86. Levine, R. S. et al. Ecological niche and geographic distribution of human monkeypox in Africa. PLOS ONE 2, e176 (2007).

87. Kearney, M., Simpson, S. J., Raubenheimer, D. \& Helmuth, B. Modelling the ecological niche from functional traits. Phil. Trans. R. Soc. B Biol. Sci. 365, 3469-3483 (2010).

88. Plowright, R. K et al. Transmission or within-host dynamics driving pulses of zoonotic viruses in reservoir-host populations. PLoS Negl. Trop. Dis. 10, e0004796 (2016)

89. Amman, B. R. et al. Seasonal pulses of Marburg virus circulation in juvenile Rousettus aegyptiacus bats coincide with periods of increased risk of human infection. PLoS Pathog. 8, e1002877 (2012)

90. Pigott, D. M. et al. Mapping the zoonotic niche of Marburg virus disease in Africa. Trans. $R$. Soc. Trop. Med. Hyg. 109, 366-378 (2015).

91. Brierley, L., Vonhof, M., Olival, K., Daszak, P. ₹ Jones, K. Quantifying global drivers of zoonotic bat viruses: a process-based perspective Am Nat 187 E53-E64 (2016).

92. Ksiazek, T. G. et al. A novel coronavirus associated with severe acute respiratory syndrome. N. Engl. J. Med. 348, 1953-1966 (2003).

93. Hutin, Y. et al. Outbreak of human monkeypox Democratic Republic of Congo, 1996 to 1997. Emerg. Infect. Dis. 7, 434 (2001).

94. Li, Q. et al. Epidemiology of human infections with avian influenza A (H7N9) virus in China. N. Engl. J. Med 370, 520-532 (2014).

95. Hayes, E. B. et al. Epidemiology and transmission dynamics of West Nile virus disease. Emerg. Infect. Dis. 11, 1167-1173 (2005)

96. Baize, S. et al. Emergence of Zaire Ebola virus disease in Guinea - preliminary report. N. Engl. J. Med. 371 , 1418-1425 (2014)

97. Saéz, A. M. et al. Investigating the zoonotic origin of the West African Ebola epidemic. EMBO Mol. Med. 7 17-23 (2015).

98. Kieft, R. et al. Mechanism of Trypanosoma brucei gambiense (group 1) resistance to human trypanosome lytic factor. Proc. Natl Acad. Sci. USA 107, 16137-16141 (2010)

99. Simarro, P. P. et al. Estimating and mapping the population at risk of sleeping sickness. PLoS Negl. Trop. Dis. 6, e1859 (2012).

100. Jezek, Z. \& Fenner, F. in Monographs in Virology Vol. 17 (ed. Melnick, J. L.) 119-121 (Karger, 1988)

101. Anthony, S. J. et al. A strategy to estimate unknown viral diversity in mammals. mBio 4, e00598-13 (2013).

This study estimates the number of viruses from nine viral families in one bat host, and uses that to extrapolate and estimate that there would be 320,000 viruses from those families in mammals.

102. Temmam, S., Davoust, B., Berenger, J.-M., Raoult, D. $\&$ Desnues, C. Viral metagenomics on animals as a tool for the detection of zoonoses prior to human infection? Int. J. Mol. Sci. 15, 10377-10397 (2014).

103. Hoffmann, M. et al. The impact of conservation on the status of the world's vertebrates. Science 330 1503-1509 (2010)

104. Ley, R. E. et al. Evolution of mammals and their gut microbes. Science 320, 1647-1651 (2008).

105. Turnbaugh, P. J. et al. The human microbiome project: exploring the microbial part of ourselves in a changing world. Nature 449, 804 (2007).

106. Dobson, A., Lafferty, K. D. Kuris, A. M. Hechinger, R. F. \& Jetz, W. Homage to Linnaeus: how many parasites? How many hosts? Proc. Natl Acad. Sci. USA 105, 11482-11489 (2008).

107. Heesterbeek, H. et al. Modeling infectious disease dynamics in the complex landscape of global health. Science 347, aaa4339 (2015).

108. Haas, C. N., Rose, J. B. \& Gerba, C. P. Quantitative Microbial Risk Assessment (John Wiley \& Sons, 2014).

109. Mitscherlich, E. \& Marth, E. H. Microbial Survival in the Environment: Bacteria and Rickettsiae Importan in Human and Animal Health (Springer Science \& Business Media, 2012)

110. Silva, E. F. et al. Characterization of virulence of Leptospira isolates in a hamster model. Vaccine $\mathbf{2 6}$ 3892-3896 (2008).
111. Elmore, S. A et al. Toxoplasma gondii: epidemiology, feline clinical aspects, and prevention. Trends Parasitol. 26, 190-196 (2010)

112. Dubey, J. Toxoplasma gondii oocyst survival under defined temperatures. J. Parasitol. 84, 862-865 (1998).

113. Jones, J. \& Dubey, J. Waterborne toxoplasmosis recent developments. Exp. Parasitol. 124, 10-25 (2010)

114. Leroy, E. M. et al. Fruit bats as reservoirs of Ebola virus. Nature 438, 575-576 (2005).

115. Pourrut, X. et al. Spatial and temporal patterns of Zaire ebolavirus antibody prevalence in the possible reservoir bat species. J. Infect. Dis. 196, S176-S183 (2007).

116. Prescott, J. et al. Postmortem stability of Ebola virus. Emerg. Infect. Dis. 21, 856 (2015).

117. Leroy, E. M. et al. Human Ebola outbreak resulting from direct exposure to fruit bats in Luebo, Democratic Republic of Congo, 2007. Vector Borne Zoonotic Dis. 9, 723-728 (2009).

118. Leroy, E. M. et al. Multiple Ebola virus transmission events and rapid decline of central African wildlife. Science 303, 387-390 (2004).

119. Judson, S., Prescott, J. \& Munster, V. Understanding Ebola virus transmission. Viruses 7, 511-521 (2015)

\section{Acknowledgements}

The authors thank J. Wood and E. Fleishman for helpfu comments and conversations. R.K.P. and H.M. are supported by the Commonwealth of Australia, the State of New South Wales and the State of Queensland under the National Hendra Virus Research Program, awarded through the Rural Industries Research and Development Corporation. R.K.P is supported by the US National Institutes of General Medical Sciences IDeA Program (grants P20GM103474 and P30GM110732), P. Thye, the Morris Animal Foundation, Montana University System Research Initiative (grant 51040-MUSRI2015-03), a Defense Advanced Research Projects Agency (DARPA) Young Faculty Award and the US Department of Defense Strategic Environmental Research and Development Program (SERDP; grant RC-2633). J.O.L.-S. is supported by the US National Science Foundation (NSF; grants OCE-1335657 and DEB-1557022) and the US Department of Defense SERDP (grant RC-2635). J.O.L.-S., A.L.G. and P.J.H. are supported by the RAPIDD program of the Science $\delta$ Technology Directorate of the Department of Homeland Security, the Fogarty International Center (part of the US National Institutes of Health), and by IDEAS (Infectious Disease Evolution Across Scales), which is a Research Coordination Network (DEB-1354890) funded by the US National Science Foundation.

Competing interests statement

The authors declare no comparing interests.

Publisher's note

Springer Nature remains neutral with regard to jurisdictional claims in published maps and institutional affiliations.

\section{SUPPLEMENTARY INFORMATION}

See online article: $\underline{\mathrm{S} 1}$ (box) | $\underline{\mathrm{S} 2}$ (movie)

ALL LINKS ARE ACTIVE IN THE ONLINE PD 